\title{
Deposition of Transparent Boron Nitride Thin Film in a Microwave Discharge
}

\author{
Osamu MATSUMOTO*, Chikara InAGAKI and Haruo Uyama
}

Received February 24, 1988 ; Accepted April 13, 1988

\begin{abstract}
Transparent boron nitride thin films were deposited on fused silica and silicon substrates by the reaction of $\mathrm{B}_{2} \mathrm{H}_{6}$ with nitrogen in a microwave discharge. The deposit by the discharge for $1 \mathrm{~h}$ was identified as hexagonal BN by IR, XPS, and X-ray diffraction. The film deposited on the fused silica substrate was transparent and the $a b-$ sorption was observed from $260 \mathrm{~nm}$.
\end{abstract}

\section{INTRODUCTION}

The absorption of $\mathrm{BN}$ film, which was prepared by chemical vapor deposition onto fused silica using the reaction of $\mathrm{BCl}_{3}$ with $\mathrm{NH}_{3}$ at $600-1100^{\circ} \mathrm{C}$, was measured (1). The main absorption peak was at about $6.2 \mathrm{eV}$ and sharp drop occurred near $5.6 \mathrm{eV}$. A plasma assisted chemical vapor deposition using several boron compounds and $\mathrm{NH} 3$ as reactants has been applied to prepare $\mathrm{BN}$ thin films $(2,3)$. In this case, deposits were generally amorphous. An amorphous BN film which was transparent in visible region was prepared out of the discharge region in the microwave discharge. However, the IR spectroscopy was only applied to identify the deposited film (4).

We have studied the formation of diamond like carbon film using microwave discharge and clarified the deposition mechanism of diamond like carbon film by surface analyses of the deposit together with plasma diagnostics $(5,6)$. The deposition of BN films in the same procedure of diamond like carbon film deposition has been carried out and the transparent BN thin film has been formed. In the present paper, the optical absorption of the BN thin film from $\mathrm{B}_{2} \mathrm{H}_{6}-\mathrm{N}_{2}-$ Ar plasma prepared with microwave discharge is reported together with the characterization of the film by IR, X-ray diffraction, and XPS analyses.

\section{EXPERIMENTAL}

\subsection{Materials}

A fused silica plate of $1 \mathrm{~mm}$ thickness and (111) oriented silicon wafer of 400 $\mu \mathrm{m}$ thickness were used as substrates. Dimension of samples was $10 \mathrm{~mm} \times 10 \mathrm{~mm}$ in each case. The substrates were chemically cleaned by degreasing in acetone. Moreover, the silicon wafer was chemically etched in HF solution as described in a previous paper (5). Purified. $\mathrm{B}_{2} \mathrm{H}_{6}(1 \%)-\mathrm{N}_{2}$ mixture gas and argon were used as plasma gases.

\subsection{Apparatus and Experimental Procedures}

The apparatus for the preparation of BN films is approximately same with that used in the deposition of diamond like carbon (5) as schematically shown in Fig. 1. Microwave power at a frequency of $2450 \mathrm{MHz}$ was transferred into the fused silica discharge tube through a rectangular wave guide from a $1 \mathrm{~kW}$ magnetron and the continuous discharge was maintained. The substrates were set on a fused silica boat placed at the desired position in the discharge tube. After the evacuation in the discharge tube below $5 \times 10^{-4}$ Torr, $\mathrm{B}_{2} \mathrm{H}_{6}(1 \%)-\mathrm{N}_{2}$ mixture gas was introduced into the discharge tube with argon at a ratio of $2: 1$ from each cylinder through flow meters. The flow rate of the gas mixture and the total

\section{Department of Chemistry, College of Science and Engineering, Aoyama Gakuin} University (Chitosedai, Setagaya-ku, Tokyo 157)

Key words: Boron nitride, Microwave discharge 
pressure in the discharge tube were $15 \mathrm{sccm}$ and 1 Torr, respectively. The power supplied was $100 \mathrm{~W}$. The surface temperature of substrates during discharge was measured using a thermocrayon and $\mathrm{CA}$ thermocouples. After the deposition of BN films for $1 \mathrm{~h}$, the deposits were characterized by IR spectroscopy, XPS, and $\mathrm{X}$-ray diffraction. The absorption spectra of the films were measured in visible and near UV regions to compare the optical properties with those of the films prepared by the other method.

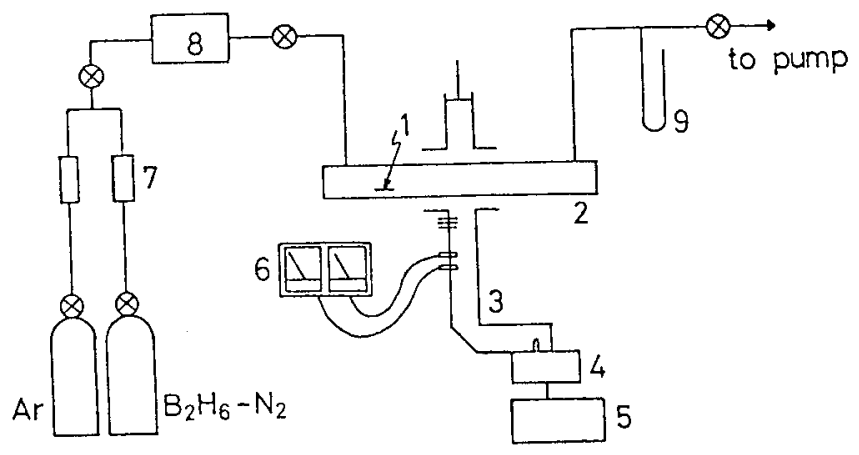

Fig. 1 Reaction apparatus

1. substrate, 2. discharge tube, 3. wave guide,

4. magnetron, 5. DC power source, 6. watt meter,

7. flow meter, 8. mass flow controller, 8. manometer

\section{RESULTS AND DISCUSSION}

3.1 Relation between surface temperature of the substrate and deposits

The silicon wafer and the fused silica plate were set in the several parts in the discharge tube. The surface temperature of the substrates measured with thermocrayon and thermocouples are given in Table 1 . The temperature on the silicon substrate was slightly higher than that on the fused silica substrate. In the center of the cavity, the surface temperature of the substrate was higher than $1000^{\circ} \mathrm{C}$. When the substrate was set in the upper stream of the gas flow, the temperature decreased with increasing distance from the center of the cavity.

Table 1 surface temperature of the substrate set in several parts in the discharge tube

\begin{tabular}{cccc}
\hline Distance from the center & \multicolumn{2}{c}{ Surface temperature } \\
\cline { 2 - 3 } of the cavity $(\mathrm{cm})$ & Si wafer & Fused silica \\
\hline 0 & $>1000$ & $>1000$ \\
4 & 1000 & 1000 \\
8 & 700 & 650 \\
16 & 420 & 360 \\
20 & 310 & 290 \\
25 & 200 & 180 \\
\hline
\end{tabular}

In the center of the cavity, the decomposition of the deposit to $B$ and $N$ was observed by XPS as shown in Fig. 2. With increasing distance from the center of the cavity, the decomposition of BN was suppressed. When the substrate was set at $25 \mathrm{~cm}$ upper stream of the gas flow from the center of the cavity, the surface temperature was about $200^{\circ} \mathrm{C}$ and $\mathrm{BN}$ was only detected by XPS. Thus the results obtained on the deposits on the substrates at $25 \mathrm{~cm}$ is discussed.

\subsection{Characterization of deposits}

The deposits on the fused silica plate and the silicon wafer after the discharge for $1 \mathrm{~h}$ were characterized by several methods.

(1) IR absorption spectra: The IR absorption spectra of the deposit on the 
silicon wafer are shown in Fig. 3. The absorption bands at $1400 \mathrm{~cm}^{-1}$ and 800 $\mathrm{cm}^{-1}$ which were characteristic in the spectra of hexagonal graphite type BN were identified (7). Peaks at about $1100 \mathrm{~cm}^{-1}$ and $600 \mathrm{~cm}^{-1}$ which were attributed to Si were also identified. The peaks at $1080 \mathrm{~cm}^{-1}$ due to cubic BN (8) was not identified in the deposit.

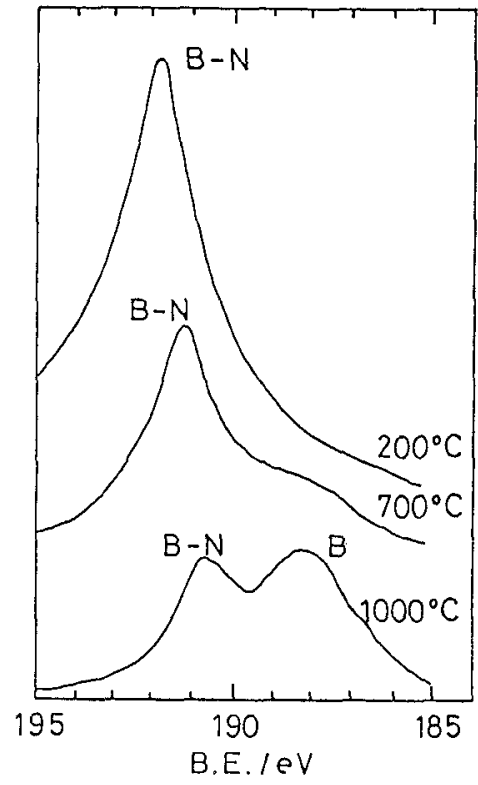

Fig. 2 XPS spectra of B1s for the deposits on the silicon substrates at several temperatures.

(2) XPS spectra: XPS spectra of the deposits on both substrates are shown in Fig. 4. The similar peaks were observed in the deposits on the both substrates. The peaks of both B1s and N1s electrons are comparable with those of hexagonal graphite type BN powder. As the peaks of Si2p electron from $\mathrm{Si}$ or $\mathrm{SiO}_{2}$ which was the substrate were not identified, the deposited film would be considerably thick.

(3) X-ray diffraction: The deposits were amorphous by a normal X-ray diffraction method. As the thickness of the deposit was estimated to be $0.3 \mathrm{um}$ from the measurement of the weight gain by the deposition, a thin film X-ray diffraction method was applied. The $\mathrm{X}$-ray diffraction pattern of the deposit on the fused silica substrate is shown in

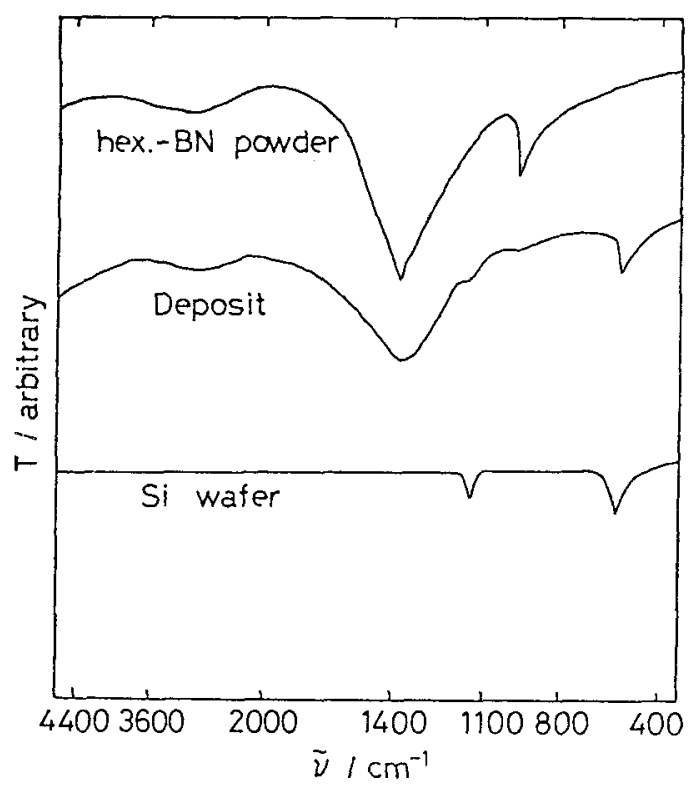

Fig. 3 IR spectra of deposits on the silicon substrates together with those of silicon wafer only and hexagonal graphite type BN powder.

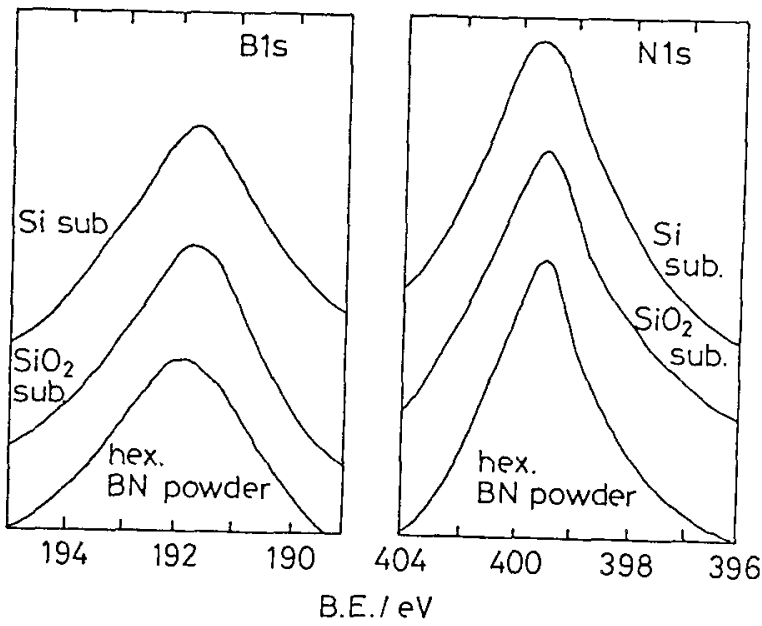

Fig. 4 XPS spectra of B1s and N1s electrons for the deposits on the several substrates. 
Fig. 5. The diffraction peaks at about $26.3^{\circ}$ and $42.2^{\circ}$ of $2 \theta$ were identified by the irradiation of CuK $_{\alpha}$ Iine. These peaks due to (002) and (100) planes of hexagonal graphite type $\mathrm{BN}$, respectively.

As the results of the characterizations mentioned above, the deposit by the microwave discharge of $\mathrm{B}_{2} \mathrm{H}_{6}-\mathrm{N}_{2}-\mathrm{Ar}$ mixture gas was identified as a thin film of hexagonal graphite type BN.

\subsection{Absorption of BN thin film}

The absorption of the transparent thin film deposited on the fused silica plate from the microwave discharge of $\mathrm{B}_{2} \mathrm{H}_{6}-\mathrm{N}_{2}-\mathrm{Ar}$ mixture gas was measured at room temperature for wave length between 700 $\mathrm{nm}$ and $200 \mathrm{~nm}$. The transmission curve is shown in Fig. 6 with the curve of fused silica plate. The deposit showed the sharp drop of transmittance at about $260 \mathrm{~nm}$. The sharp drop is attributed to the optical band gap of $5.8 \mathrm{eV}$ of $\mathrm{BN}$ thin film (1).

The present work was partly supported by a Grant-in-Aid for Scientific Research No. 62470068 from the Ministry of Education, Science, and Culture.

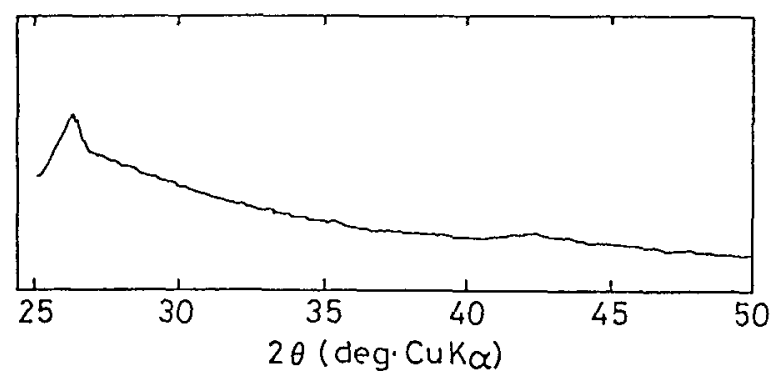

Fig. 5 Thin film X-ray diffraction pattern of the deposit on the fused silica substrate.

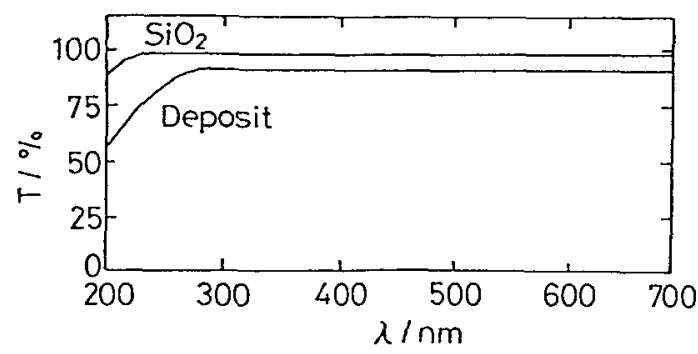

Fig. 6 Transmittance variation with wave length of deposits on the fused silica substrate together with that of fused silica.

\section{REFERENCES}

1) M. Sano and M. Aoki, Thin Solid Films, 83, 247 (1981).

2) W. Schnolla and H. L. Hartnagel, Solid State Electronics, 26, 931 (1983).

3) O. Gafli, A. Grili, D. Itzhak, A. Inspektor, and R. Avni, Thin Solid Films, $72,532(1980)$.

4) K. Montasser, T. Tamano, S. Hattori, and S. Morita, Plasma Chem. Plasma, Process. 4, 251 (1984).

5) O. Matsumōto, H. Toshima, and Y. Kanzaki, Thin Solid Films, 128, 785 (1985).

6) 0 . Matsumoto and T. Katagiri, Thin Solid Films, 146283 ( $198 \overline{7}$.

7) T. Takahashi, H. Itoh, and A. Takeuchi, J. Cryst. Growth, 47, 245 (1979).

8) P. J. Gelisse, S. S. Mitra, J. N. Plendl, R. D. Griffis, C. Mansur, R. Marshall, and E. A. Pascoe, Phys. Rev., 155, 1039 (1967). 